\title{
The Cut-off Value of Blood Mercury Concentration in Relation to Insulin Resistance
}

\author{
Seok-Hoon Lee', Beomhee Choi², Soo-Jung Park', Young-Sang Kim³, Nam-Seok Joo ${ }^{1, *}$ \\ ${ }^{1}$ Department of Family Practice and Community Health, Ajou University School of Medicine, Suwon; ${ }^{2}$ CHA Anti-aging Institute, CHA University, Seoul; ${ }^{3}$ Department \\ of Family Medicine, CHA University, CHA Bundang Medical Center, Seongnam, Korea
}

Background: Increased blood mercury concentration is associated with inflammation, and chronic inflammation can cause insulin resistance. We examined the cut-off value of blood mercury in relation to an increased score on the homeostasis model assessment for insulin resistance (HOMA-IR).

Methods: We used data from the Korean National Health and Nutrition Examination Survey (2008-2010). Relevant data from 5,184 subjects (2,523 men and 2,661 women) were analyzed cross-sectionally. General linear analysis was performed to evaluate the relationship between HOMA-IR score and blood mercury concentration. In addition, we determined the cut-off value of blood mercury concentration in relation to increased HOMA-IR score (> 2.34) using an ROC curve.

Results: The mean value of blood mercury concentration in men and women was $5.88 \mu \mathrm{g} / \mathrm{L}$ and $4.11 \mu \mathrm{g} / \mathrm{L}$, respectively. In men, comparing to the first quartile, HOMA-IR score increased significantly in the third and fourth blood mercury quartiles. In women, however, the increase in HOMA-IR score was not significant. The cut-off value that best represented the association between increased HOMA-IR score and blood mercury concentration in men was found to be $4.71 \mu \mathrm{g} / \mathrm{L}$.

Conclusion: Blood mercury concentration was associated with increased HOMA-IR score in men, and the cut-off value of blood mercury concentration that was correlated with increased HOMA-IR score was around $4.71 \mu \mathrm{g} / \mathrm{L}$.

Received March 29, 2017

Reviewed May 18, 2017

Accepted July 4, 2017

*Corresponding author

Nam-Seok Joo

(iD)

http://orcid.org/0000-0001-5895-1800

Department of Family Practice and Community Health, Ajou University School of Medicine, 164 Worldcup-ro, Youngtong-gu, Suwon 16499, Korea Tel: $+82-31-219-5324$

Fax: +82-31-219-5218

E-mail: jchcmc@hanmail.net

Key words: Mercury, HOMA-IR, Insulin resistance, Cut-off value, Diabetes

\section{INTRODUCTION}

The prevalence of type 2 diabetes is increasing. Globally, it was estimated that 415 million people aged $20-79$ years had diabetes in 2015. ${ }^{1}$ People with diabetes confront numerous serious health problems, including blindness, kidney failure, cardiovascular disease, premature death, cognitive decline, and amputations. ${ }^{2}$ A number of lifestyle factors are known to affect development of type 2 diabetes, including lack of physical activity, obesity, a dietary shift toward more refined carbohydrates, urbanization, and stress. ${ }^{3,4}$ At the cellular level, decreased beta $(\beta)$ cell function and increased insulin resistance are known to play a part in the pathogenesis of type
2 diabetes. ${ }^{5}$ Pancreatic $\beta$ cells, which intrinsically possess low antioxidant enzyme activity, are vulnerable to oxidative damage. ${ }^{6}$ Indeed, oxidative stress gives rise to $\beta$ cell $\operatorname{loss}^{7}$ and is known to be involved in diabetes. $^{8}$

Blood mercury results in cellular production of reactive oxygen species and lipid peroxidation. ${ }^{9}$ Blood mercury also inhibits the activity of mitochondrial enzymes, leading to mitochondrial membrane depolarization and damage, which also increase the production of reactive oxygen species. ${ }^{10}$ Human exposure to mercury, especially methyl mercury, occurs through various pathways, including consumption of seafood, occupational and household use of products containing mercury, and use of dental amalgams. ${ }^{11}$ In par- 
ticular, high dietary intake of seafood is one of the most effective routes of exposure. ${ }^{12}$ The adverse health effects of mercury are a result of the extent of exposure (quantity, frequency, and duration), the speciation of mercury accumulated in the body, and the age of the person. ${ }^{13}$ Unfortunately, the human body has no capability for active excretion of mercury. Therefore, mercury accumulates in the tissues of the human body. ${ }^{14}$ This accumulation is associated with chronic inflammation and the development of many diseases, such as stroke ${ }^{15}$, hypertension, and insulin resistance. ${ }^{16}$ Therefore, increased mercury exposure could contribute to the pathology of diabetes due to oxidative stress, which damages pancreatic $\beta$ cells and has effects on glucose tolerance and insulin secretion..$^{17,18}$

The homeostasis model assessment for insulin resistance (HOMA-IR) has been widely used to estimate the extent of insulin resistance. The cutoff value for HOMA-IR in Korean non-diabetic adults has been reported as 2.34 (sensitivity, 62.8\%; specificity, 65.7\%). ${ }^{19}$ Furthermore, previous studies demonstrated that blood mercury concentration was associated with HOMA-IR score in Koreans. ${ }^{20}$ However, the cut-off value of blood mercury concentration that enables prediction of increasing HOMA-IR score has not been established. Therefore, in this study, we tried to pinpoint the cut-off value for blood mercury concentration that has the highest sensitivity and specificity for predicting an increase in HOMA-IR score.

\section{METHODS}

\section{Study data}

The Korea National Health and Nutrition Examination Survey (KNHANES) is a nationwide cross-sectional health survey. Participants are representatives of the Korean population. KNHANES results have external validity. This study was based on data obtained from the fourth (2008 and 2009, IV-2, 3) to fifth (2010, V-1) years of the KNHANES. From an initial total of 29,235 subjects, 23,618 were excluded due to missing data on blood mercury $(22,883$ subjects), age $<19$ or $\geq 70$ years (10,474 subjects), type 2 diabetes (422 subjects), or an extreme blood mercury level (>500 $\mu \mathrm{g} / \mathrm{L}$, one subject). Diabetes was defined as current use of anti-diabetic medications, a self-reported physician's diagnosis of diabetes, or a fasting glucose level $\geq 126 \mathrm{mg} / \mathrm{dL}(6.99 \mathrm{mmol} / \mathrm{L})$. A final total of 5,184 subjects $(2,523$ men and 2,661 women) were included in

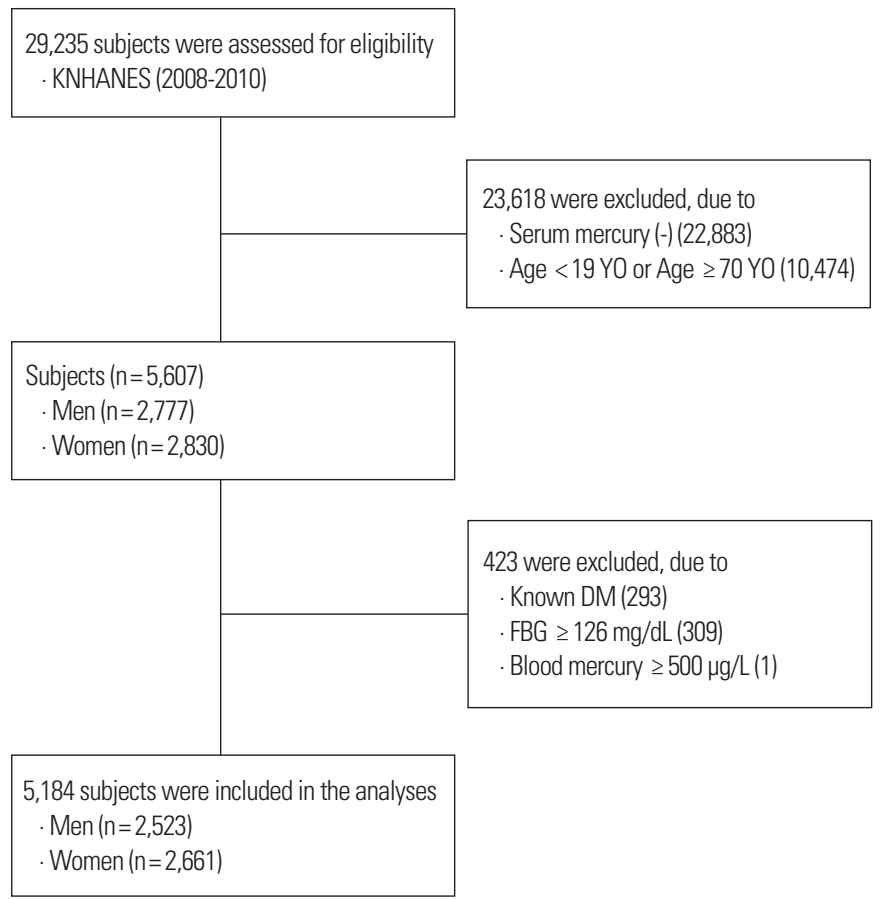

Figure 1. Flow chart of study subjects. KNHANES, Korea National Health and Nutrition Examination Survey; YO, years old; DM, type 2 diabetes; FBG, fasting blood glucose.

this study (Fig. 1), consisting of 830 men and 885 women in 2008, 845 men and 847 women in 2009, and 848 men and 929 women in 2010, suggesting that the data are still representative of the population as a whole. All participants provided written informed consent to participate in the survey. The Institutional Review Board of Ajou University Hospital (Suwon, Republic of Korea) approved this study (AJIRB-MED-EXP-16-483).

\section{Measurements}

Blood mercury was measured by the Gold-Amalgam method using a DMA-80 apparatus (Milestone, Italy); the inter-assay coefficients of variation were $0.47-6.08 \%$. Serum insulin concentrations were measured using an INS-Irma gamma counter with an immunoradiometric assay (Biosource, Nivelles, Belgium), and blood glucose concentrations were measured using a Pureauto S GLU automated analyzer with an enzymatic assay (Daiichi, Tokyo, Japan). Insulin resistance was estimated using HOMA-IR score calculated as [fasting insulin $(\mu \mathrm{IU} / \mathrm{mL}) \times$ fasting glucose $(\mathrm{mg} / \mathrm{dL})] / 405$. Physical examinations were performed by a trained examiner who followed a standardized procedure. Current smokers were defined 
as individuals who had smoked more than five packs of cigarettes during their lifetime and were currently smoking; non-smokers had no history of smoking; past-smokers included smokers who had smoked in the past but had quit. Regular alcohol drinkers included those who currently drank alcohol more than one time per month, while nondrinkers comprised all others. Physical activity was assessed by a questionnaire and categorized as 'yes' or 'no.' A 'yes' indicated 30 minutes of moderate physical activity three or more times in the last week that made the subject more tired than usual. Nutrient intake including total caloric intake was assessed with a 24 hours dietary recall questionnaire administered by a trained dietician. Results were calculated using the Food Composition Table developed by the National Rural Resources Development Institute (7th revision). Age at menarche was determined by a health questionnaire administered by a trained examiner. Women were classified into premenopausal, menopausal women and women on hormone replacement therapy.

\section{Statistics}

Complex sample analysis was used to weight the KNHANES data following the guidance on statistics from the Korea Centers for Disease Control and Prevention. General characteristics of the study subjects were age; blood pressure; body mass index; waist circumference; and metabolic markers of total cholesterol, triglyceride, high-density lipoprotein cholesterol level, fasting blood glucose, insulin, and blood mercury level and were analyzed by a simple descriptive method after data weighting. Alcohol consumption and smoking status were evaluated by the $\chi^{2}$ test. To evaluate the relationship between blood mercury concentration and HOMA-IR score, blood mercury concentration was divided into quartiles after $\log$ transformation to create a normal distribution. Linear regression analysis was conducted in men and women after adjusting for age, physical activity, alcohol intake, smoking status, and daily total energy intake. For women, we also adjusted for oral contraceptive intake, menopause status, and hormone replacement therapy. Receiver operating characteristic curves and cut-off values for blood mercury concentrations associated with increased HOMA-IR were calculated. The $P$ values were used to assess the significance of all analyses. Data were analyzed using SPSS 20.0 (IBM Corp., Armonk, NY, USA) to account for the complex sampling design.
Table 1. General characteristics of the study subjects

\begin{tabular}{|c|c|c|c|}
\hline Variables & Men $(n=2,523)$ & Women $(n=2,661)$ & $P$ \\
\hline Age (yr) & $38.7(0.3)$ & $40.0(0.3)$ & $<0.001$ \\
\hline Height (cm) & $171.4(0.2)$ & $158.6(0.1)$ & $<0.001$ \\
\hline Weight (kg) & $70.6(0.3)$ & $57.1(0.2)$ & $<0.001$ \\
\hline $\mathrm{BMI}\left(\mathrm{kg} / \mathrm{m}^{2}\right)$ & $24.0(0.8)$ & $22.7(0.8)$ & $<0.001$ \\
\hline WC (cm) & $83.4(0.2)$ & $76.2(0.2)$ & $<0.001$ \\
\hline $\mathrm{SBP}(\mathrm{mmHg})$ & $116.0(0.4)$ & $110.0(0.4)$ & $<0.001$ \\
\hline $\mathrm{DBP}(\mathrm{mmHg})$ & $77.1(0.3)$ & $71.6(0.2)$ & $<0.001$ \\
\hline TC (mg/dL) & $185.4(1.0)$ & $184.9(0.9)$ & 0.711 \\
\hline TG (mg/dL) & $149.8(3.1)$ & $103.2(1.7)$ & $<0.001$ \\
\hline $\mathrm{HDLC}$ (mg/dL) & $50.0(0.3)$ & $57.0(0.3)$ & $<0.001$ \\
\hline FBS (mg/dL) & $92.7(0.2)$ & $90.6(0.2)$ & $<0.001$ \\
\hline Insulin ( $\mu \mid \mathrm{U} / \mathrm{mL})$ & $10.1(0.1)$ & $10.0(0.1)$ & 0.789 \\
\hline HOMA-IR & $2.33(0.1)$ & $2.27(0.1)$ & 0.159 \\
\hline Energy (kcal/day) & 2,394.0 (25.5) & 1,684.8 (15.3) & $<0.001$ \\
\hline Blood mercury ( $\mu \mathrm{g} / \mathrm{L})$ & $5.88(0.11)$ & $4.11(0.07)$ & $<0.001$ \\
\hline \multicolumn{4}{|l|}{ Smoking } \\
\hline Yes, n (\%) & $1,173(46.5)$ & $158(5.9)$ & $<0.001$ \\
\hline \multicolumn{4}{|l|}{ Alcohol } \\
\hline Yes, n (\%) & $1,940(76.9)$ & $1,152(43.3)$ & $<0.001$ \\
\hline \multicolumn{4}{|l|}{ Physical activity } \\
\hline Yes, $n(\%)$ & $360(14.3)$ & $361(13.6)$ & 0.043 \\
\hline
\end{tabular}

Values are presented as mean (standard error) after data weighting.

$\mathrm{BMI}$, body mass index; WC, waist circumference; SBP, systolic blood pressure; DBP, diastolic blood pressure; TC, total cholesterol; TG, triglycerides; HDLC, high-density lipoprotein cholesterol; FBS, fasting blood sugar; HOMA-IR, homeostasis model assessment for insulin resistance; Energy, total daily energy intake; Blood mercury, methyl-mercury.

\section{RESULTS}

A total of 5,184 subjects' data were analyzed in this study. The general characteristics of the study subjects are summarized in Table 1. The mean HOMA-IR score was 2.33 for men and 2.27 for women. The mean body mass index (BMI) and waist circumference were $24.0 \mathrm{~kg} / \mathrm{m}^{2}$ and $83.4 \mathrm{~cm}$ in men and $22.7 \mathrm{~kg} / \mathrm{m}^{2}$ and $76.2 \mathrm{~cm}$ in women, respectively. Other respective values in men and women were as follows: average fasting blood sugar (92.7 and $90.6 \mathrm{mg} / \mathrm{dL})$, insulin (10.1 and $10.0 \mu \mathrm{IU} / \mathrm{mL})$, total cholesterol (185.4 and $184.9 \mathrm{mg} / \mathrm{dL}$ ), high-density lipoprotein cholesterol (50.0 and $57.0 \mathrm{mg} / \mathrm{dL}$ ), and triglyceride (149.8 and $103.2 \mathrm{mg} /$ $\mathrm{dL})$. The average blood mercury level in men $(5.88 \mu \mathrm{g} / \mathrm{L})$ was higher than that in women $(4.11 \mu \mathrm{g} / \mathrm{L})$. As expected, the proportion of current smokers and regular alcohol consumption were higher in men than in women.

After blood mercury concentration was log-transformed, we di- 
Table 2. HOMA-IR score by serum logHg quartile

\begin{tabular}{lcclcc}
\hline \multicolumn{3}{c}{ Men } & & \multicolumn{2}{c}{ Women } \\
\cline { 1 - 2 } \cline { 5 - 6 } Quartile (range) & HOMA-IR & & Quartile (range) & HOMA-IR \\
\hline $01(0.68-3.54)$ & $2.20(0.05)$ & & $01(0.76-2.54)$ & $2.20(0.48)$ \\
$02(3.55-5.13)$ & $2.25(0.49)$ & & $02(2.55-3.56)$ & $2.23(0.55)$ \\
$03(5.14-7.41)$ & $2.45(0.94)^{*}$ & & $03(3.57-4.94)$ & $2.34(0.57)$ \\
$04(7.42-56.97)$ & $2.45(0.89)^{\dagger}$ & & $04(4.95-33.93)$ & $2.30(0.57)$ \\
$P$ for trend & 0.03 & & $P$ for trend & 0.22 \\
\hline
\end{tabular}

Values are presented as mean (standard error) after data weighting.

$P$ for trend was obtained by a general linear model after adjustment for age, moderate physical activity, smoking status, alcohol intake, and total energy intake in men and further adjustment for menopausal status, intake of oral contraceptives, and hormone replacement therapy in women.

${ }^{*} P<0.05$ in the comparison between 01 and 03 .

${ }^{\dagger} P<0.05$ in the comparison between 01 and 04 .

HOMA-IR, homeostasis model assessment for insulin resistance.

vided it into quartiles (Table 2). HOMA-IR score showed a gradual increase as blood mercury quartile increased in men after adjusting for age, moderate physical activity, smoking status, alcohol intake, and daily total energy intake. In men, the average HOMA-IR score increased by 0.25 when the highest blood mercury quartile was compared to the lowest blood mercury quartile. In women, after adjusting for covariates, there was no significant difference in HOMA-IR score between quartiles.

The area under the curve (AUC) value of mercury associated with increased HOMA-IR score in men was 0.534 (95\% CI, 0.5110.558) (Table 3). The cut-off value of blood mercury concentration corresponding to the AUC associated with an increased HOMA-IR score was $4.71 \mu \mathrm{g} / \mathrm{L}$ (Fig. 2).

\section{DISCUSSION}

This cross-sectional study examined blood mercury concentration in relation to HOMA-IR score using KNHANES data from 2008 to 2010. In our study, we observed consistent increases in HOMA-IR score with increasing blood mercury. HOMA-IR score was significantly higher in the third and fourth quartiles of blood mercury than in the first quartile in men. A blood mercury level over $4.71 \mu \mathrm{g} / \mathrm{L}$ was significantly associated with a significant increase in HOMA-IR score; this could be a cut-off value for blood mercury concentration in Korean non-diabetic men.

Oxidative stress plays a role in the progression of pancreatic $\beta$-cell dysfunction and insulin resistance. ${ }^{21}$ Shenker et al. ${ }^{22}$ revealed
Table 3. AUC and its cut-off value for blood mercury concentration according to an increase of HOMA-IR score in men

\begin{tabular}{cccccc}
\hline & AUC $(95 \% \mathrm{Cl})$ & $\mathrm{Hg}(\mu \mathrm{g} / \mathrm{L})$ & Sensitivity & 1-Specificity & $P$ \\
\hline HOMA-IR & $0.534(0.511-0.558)$ & 4.71 & 0.608 & 0.547 & 0.004 \\
\hline
\end{tabular}

$\mathrm{Hg}$ represents the blood mercury concentration corresponding to the cut-off value. AUC, area under the curve; HOMA-IR, homeostasis model assessment for insulin resistance.

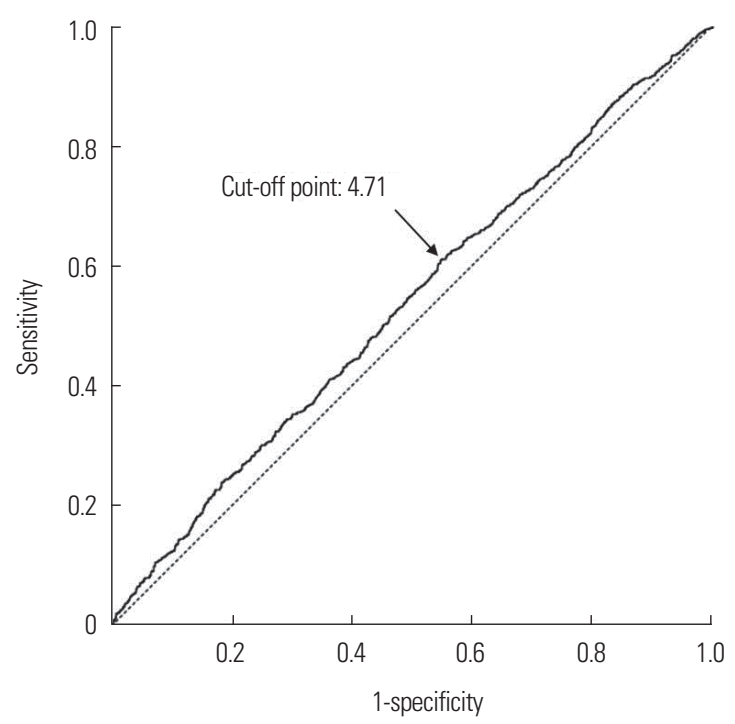

Figure 2. Receiver operating characteristic (ROC) curve of blood mercury concentration according to the presence of abnormal homeostasis model assessment for insulin resistance (HOMA-IR) score in men. This ROC curve shows the maximum association between blood mercury concentration $(4.71 \mu \mathrm{g} / \mathrm{L}, P=0.004)$ and HOMA-IR score at the point of sensitivity (0.608) and 1-specificity (0.547).

that mercury induces apoptosis in human $\mathrm{T}$ lymphocytes and hypothesized both that the target organelle is the mitochondrion and that inducing oxidative stress activates an apoptotic pathway. Their findings proposed that mercury induces oxidative stress-regulated pancreatic $\beta$-cell cytotoxicity through a mitochondrial apoptotic pathway that activates caspase- 3 in response to mitochondrial release of cytochrome c. Chen et al. ${ }^{23}$ showed that 2 or 4 weeks of oral exposure to low-dose mercury decreases plasma insulin level, increases plasma lipid peroxidation level, and elevates blood glucose and glucose intolerance. N-acetyl-1-cysteine (a ROS scavenger) prevented these mercury-induced responses. These findings demonstrate that mercury-induced oxidative stress and PI3K activation cause Akt signaling-related pancreatic $\beta$-cell dysfunction, which indicates that oxidative stress is involved in the toxic mechanism in mercury-induced hyperglycemia.

In addition, several studies have revealed that blood heavy metal 
levels are significantly associated with metabolic syndrome, including insulin resistance, after adjustment for multiple parameters. ${ }^{15,20,24}$ In this study, men who had a blood mercury concentration around $4.71 \mu \mathrm{g} / \mathrm{L}$ showed significantly higher HOMA-IR scores after adjusting for relevant confounders. Interestingly, we observed a gender difference in the association between blood mercury concentration and HOMA-IR score. Mean blood mercury concentration was higher in men than in women $(5.88 \mu \mathrm{g} / \mathrm{L}$ vs. $4.11 \mu \mathrm{g} / \mathrm{L}$ ). This result is inconsistent with the results of prior studies. ${ }^{24,25}$ In addition to the difference in blood mercury concentration, women might also respond differently to mercury exposure; in a cohort study, there was no significant association between blood mercury level and oxidative stress biomarkers in premenopausal women with low exposure levels. ${ }^{26}$ Occupational environments, fish consumption ${ }^{27}$, and frequency of smoking might cause elevated blood mercury concentration.

The mean concentration of blood mercury has gradually increased in Korean adults. This might be partially related to increased insulin resistance, which contributes to an increased risk of diabetes. ${ }^{20}$ The Korean National Environmental Health Survey (KoNEHS) revealed that the average blood mercury concentration in Koreans was greater than that of those living in European countries or in the United States in 2012 to $2014 .{ }^{28}$ In our data, average blood mercury concentration was $5.88 \mu \mathrm{g} / \mathrm{L}$ in men and $4.11 \mu \mathrm{g} / \mathrm{L}$ in women; both measurements are more than four times higher than those from individuals in the USA $(0.94 \mu \mathrm{g} / \mathrm{L})$, Canada (0.69 $\mu \mathrm{g} / \mathrm{L})$, and Germany $(0.58 \mu \mathrm{g} / \mathrm{L}){ }^{29}$

In a bio-monitoring study of cadmium, lead, and mercury concentration in the general Korean adult population, the geometric mean of serum mercury level $(3.08 \mu \mathrm{g} / \mathrm{L}, 15.35 \mathrm{nmol} / \mathrm{L})$ was more common in individuals over 40 years of age than in those younger than 40 and higher in those with more frequent fish consumption ${ }^{30}$ and alcohol consumption. Even though a blood mercury level of $3.08 \mu \mathrm{g} / \mathrm{L}$ is not notably toxic, we presume that it has an effect on insulin resistance or vascular inflammation. In addition to insulin resistance, several studies have shown positive associations between mercury exposure and higher blood pressure or hypertension ${ }^{31,32}$, mainly due to vascular inflammation. These results also support blood mercury as a risk factor for insulin resistance in the general population. In our study, the mean blood mercury concentration in men was $5.88 \mu \mathrm{g} / \mathrm{L}$. This value is higher than the lowest value in the third quartile of blood mercury concentration $(5.14 \mu \mathrm{g} / \mathrm{L})$ and the cut-off value of blood mercury for increasing HOMA-IR score $(4.71 \mu \mathrm{g} / \mathrm{L})$. This result might mean that more attention should be paid to men with lower concentrations of blood mercury so as not to increase HOMA-IR.

There are several limitations in our study. First, this was a crosssectional study, so we could not demonstrate causality between blood mercury level and insulin resistance. Second, we could not adjust for all possible confounders that might affect HOMA-IR or insulin resistance, such as $\mathrm{BMI}^{33}$ or hormones. ${ }^{34}$ Although blood mercury is associated with BMI in Koreans ${ }^{35}$, we did not adjust BMI due to multicollinerarity. Third, a personal history of fish consumption and occupational exposure are important to blood mercury concentration. However, we could not adjust for these confounders. ${ }^{36}$ In spite of these limitations, this is the first study to show the cut-off values of blood mercury concentration in connection with increased HOMA-IR score in a large population-based dataset.

In conclusion, higher blood mercury concentration is significantly associated with higher HOMA-IR score in Korean men. The cut-off value of blood mercury concentration in relation to increased HOMA-IR score was around $4.71 \mu \mathrm{g} / \mathrm{L}$ in men, which might mean that an increase in HOMA-IR score is associated with that cut-off value. Large prospective studies are needed to investigate the exact cut-off values between increased HOMA-IR score and blood mercury concentration.

\section{CONFLICTS OF INTEREST}

The authors declare no conflict of interest.

\section{REFERENCES}

1. International Diabetes Federation. IDF diabetes atlas. 7th ed. Brussels, Belgium: International Diabetes Federation; 2015 [accessed 2016 Nov 30]. Available from: URL: http://www. diabetesatlas.org

2. Goff DC Jr, Gerstein HC, Ginsberg HN, Cushman WC, Margolis KL, Byington RP, et al. Prevention of cardiovascular dis- 
ease in persons with type 2 diabetes mellitus: current knowledge and rationale for the Action to Control Cardiovascular Risk in Diabetes (ACCORD) trial. Am J Cardiol 2007;99:4i$20 \mathrm{i}$.

3. Melmed S, Polonsky KS, Larsen PR, Kronenberg HM. Williams textbook of endocrinology. 12th ed. Philadelphia: Saunders; 2011. p. 1371-435.

4. Abdullah A, Peeters A, de Courten M, Stoelwinder J. The magnitude of association between overweight and obesity and the risk of diabetes: a meta-analysis of prospective cohort studies. Diabetes Res Clin Pract 2010;89:309-19.

5. Cho JH, Kim JW, Shin JA, Shin J, Yoon KH. $\beta$-cell mass in people with type 2 diabetes. J Diabetes Investig 2011;2:6-17.

6. Lenzen S, Drinkgern J, Tiedge M. Low antioxidant enzyme gene expression in pancreatic islets compared with various other mouse tissues. Free Radic Biol Med 1996;20:463-6.

7. Robertson RP. Chronic oxidative stress as a central mechanism for glucose toxicity in pancreatic islet beta cells in diabetes. $\mathrm{J}$ Biol Chem 2004;279:42351-4.

8. Valko M, Leibfritz D, Moncol J, Cronin MT, Mazur M, Telser J. Free radicals and antioxidants in normal physiological functions and human disease. Int J Biochem Cell Biol 2007;39:44-84.

9. Stohs SJ, Bagchi D. Oxidative mechanisms in the toxicity of metal ions. Free Radic Biol Med 1995;18:321-36.

10. Carratù MR, Signorile A. Methyl mercury injury to CNS: mitochondria at the core of the matter? J Toxicol 2015;1:555551.

11. Park JD, Zheng W. Human exposure and health effects of inorganic and elemental mercury. J Prev Med Public Health 2012;45:344-52.

12. Basu N, Goodrich JM, Head J. Ecogenetics of mercury: from genetic polymorphisms and epigenetics to risk assessment and decision-making. Environ Toxicol Chem 2014;33:1248-58.

13. Clarkson TW, Magos L. The toxicology of mercury and its chemical compounds. Crit Rev Toxicol 2006;36:609-62.

14. Mercier M. International approach to the assessment of chemical risks. Sci Total Environ 1991;101:1-7.

15. Houston MC. Role of mercury toxicity in hypertension, cardiovascular disease, and stroke. J Clin Hypertens (Greenwich) 2011;13:621-7.

16. Chang JW, Chen HL, Su HJ, Liao PC, Guo HR, Lee CC. Si- multaneous exposure of non-diabetics to high levels of dioxins and mercury increases their risk of insulin resistance. J Hazard Mater 2011;185:749-55.

17. Rolo AP, Palmeira CM. Diabetes and mitochondrial function: role of hyperglycemia and oxidative stress. Toxicol Appl Pharmacol 2006;212:167-78.

18. Mahboob M, Shireen KF, Atkinson A, Khan AT. Lipid peroxidation and antioxidant enzyme activity in different organs of mice exposed to low level of mercury. J Environ Sci Health B 2001;36:687-97.

19. Lee S, Choi S, Kim HJ, Chung YS, Lee KW, Lee HC. Cutoff values of surrogate measures of insulin resistance for metabolic syndrome in Korean non-diabetic adults. J Korean Med Sci 2006;21:695-700.

20. Kim KN, Park SJ, Choi B, Joo NS. Blood mercury and insulin resistance in nondiabetic Koreans (KNHANES 2008-2010). Yonsei Med J 2015;56:944-50.

21. Evans JL, Goldfine ID, Maddux BA, Grodsky GM. Oxidative stress and stress-activated signaling pathways: a unifying hypothesis of type 2 diabetes. Endocr Rev 2002;23:599-622.

22. Shenker BJ, Guo TL, O I, Shapiro IM. Induction of apoptosis in human T-cells by methyl mercury: temporal relationship between mitochondrial dysfunction and loss of reductive reserve. Toxicol Appl Pharmacol 1999;157:23-35.

23. Chen YW, Huang CF, Tsai KS, Yang RS, Yen CC, Yang CY, et al. The role of phosphoinositide 3-kinase/Akt signaling in lowdose mercury-induced mouse pancreatic beta-cell dysfunction in vitro and in vivo. Diabetes 2006;55:1614-24.

24. Eom SY, Choi SH, Ahn SJ, Kim DK, Kim DW, Lim JA, et al. Reference levels of blood mercury and association with metabolic syndrome in Korean adults. Int Arch Occup Environ Health 2014;87:501-13.

25. Lie A, Gundersen N, Korsgaard KJ. Mercury in urine - sex, age and geographic differences in a reference population. Scand J Work Environ Health 1982;8:129-33.

26. Pollack AZ, Sjaarda L, Ahrens KA, Mumford SL, Browne RW, Wactawski-Wende J, et al. Association of cadmium, lead and mercury with paraoxonase 1 activity in women. PLoS One 2014;9:e92152.

27. You CH, Kim BG, Kim YM, Lee SA, Kim RB, Seo JW, et al. 
Relationship between dietary mercury intake and blood mercury level in Korea. J Korean Med Sci 2014;29:176-82.

28. Kim SA, Kwon Y, Kim S, Joung H. Assessment of dietary mercury intake and blood mercury levels in the Korean population: results from the Korean National Environmental Health Survey 2012-2014. Int J Environ Res Public Health 2016;13:877.

29. Ministry of Health and Welfare, Korea Centers for Disease Control and Prevention. Korea Health Statistics 2011: Korea National Health and Nutrition Examination Survey (KNHANES V-2) Seoul: Korea Centers for Disease Control and Prevention; 2012.

30. Kim NS, Lee BK. National estimates of blood lead, cadmium, and mercury levels in the Korean general adult population. Int Arch Occup Environ Health 2011;84:53-63.

31. Vupputuri S, Longnecker MP, Daniels JL, Guo X, Sandler DP. Blood mercury level and blood pressure among US women: results from the National Health and Nutrition Examination Survey 1999-2000. Environ Res 2005;97:195-200.

32. Fillion M, Mergler D, Sousa Passos CJ, Larribe F, Lemire M, Guimarães JR. A preliminary study of mercury exposure and blood pressure in the Brazilian Amazon. Environ Health 2006; $5: 29$.

33. Kang ES, Yun YS, Park SW, Kim HJ, Ahn CW, Song YD, et al. Limitation of the validity of the homeostasis model assessment as an index of insulin resistance in Korea. Metabolism 2005; 54:206-11.

34. Yeap BB, Chubb SA, Hyde Z, Jamrozik K, Hankey GJ, Flicker L, et al. Lower serum testosterone is independently associated with insulin resistance in non-diabetic older men: the Health In Men Study. Eur J Endocrinol 2009;161:591-8.

35. Bae S, Park SJ, Yeum KJ, Choi B, Kim YS, Joo NS. Cut-off values of blood mercury concentration in relation to increased body mass index and waist circumference in Koreans. J Investig Med 2016;64:867-71.

36. Kim SJ, Han SW, Lee DJ, Kim KM, Joo NS. Higher serum heavy metal may be related with higher serum gamma-glutamyltransferase concentration in Koreans: analysis of the fifth Korea National Health and Nutrition Examination Survey (KNHANES V-1, 2, 2010, 2011). Korean J Fam Med 2014; $35: 74-80$. 